\title{
Evaluation of two histological classifications for actinic keratoses - PRO classification scored highest inter-rater reliability
}

\author{
L. Schmitz, ${ }^{1, \star}$ G. Gupta, ${ }^{2,3}$ M. Stücker, ${ }^{1}$ M. Doerler, ${ }^{1}$ T. Gambichler, ${ }^{1}$ J. Welzel, ${ }^{4}$ R.M. Szeimies, ${ }^{5}$ \\ E. Bierhoff, ${ }^{6}$ E. Stockfleth, ${ }^{1}$ T. Dirschka ${ }^{7,8}$ \\ ${ }^{1}$ Department of Dermatology, Venereology and Allergology, Ruhr University, Bochum, Germany \\ ${ }^{2}$ Department of Dermatology, University Hospital Monklands, Lanarkshire, UK \\ ${ }^{3}$ School of Medicine, University of Glasgow, Glasgow, UK \\ ${ }^{4}$ Department of Dermatology and Allergology, General Hospital Augsburg, Augsburg, Germany \\ ${ }^{5}$ Department of Dermatology and Allergology, Vest Hospital, Academic Teaching Hospital University of Bochum, Recklinghausen, \\ Germany \\ ${ }^{6}$ Heinz-Werner-Seifert-Institute of Dermatopathology, Bonn, Germany \\ ${ }^{7}$ CentroDerm Clinic, Wuppertal, Germany \\ ${ }^{8}$ Faculty of Health, University Witten-Herdecke, Witten, Germany \\ *Correspondence: L. Schmitz. E-mail: I.schmitz@klinikum-bochum.de
}

\begin{abstract}
Background Actinic keratoses (AKs) can histologically be classified by the extent of atypical keratinocytes throughout the epidermis or their pattern of basal proliferation. Currently, no data on the inter-rater reliability of both scores is available. Objective To evaluate the inter-rater reliability of the two classification schemes; histological grade (AK I-III) and basal proliferation (PRO I-III).

Methods Histological images of 54 AKs were classified by 21 independent dermatopathologists with regard to basal proliferation (PRO I-III), histological grade (AK I-III) and assumed risk of progression into invasive carcinoma.

Results Overall, of the 54 AKs $16.7 \%$ (9/54) were classified as AK I, 66.7\% (36/54) as AK II, and 16.7\% (9/54) as AK III. With regards to basal growth pattern, 25.9\% (14/54) were classified as PRO I, 42.6\% (23/54) as PRO II, and 31.5\% (17/ 54) as PRO III. We observed a highly significant inter-rater reliability for PRO-grading $(P<0.001)$ which was higher than for AK-grading (Kendall's $W$ coefficient: $A K=0.488$ vs. PRO $=0.793$ ). We found substantial agreement for assumed progression risk for AKs with worsening basal proliferation $(k=0.759)$ compared to moderate agreement $(k=0.563)$ for different AK-gradings.

Conclusions Histological classification of basal growth pattern (PRO) showed higher inter-rater reliability compared to the established classification of atypical keratinocytes throughout epidermal layers. Moreover, experienced dermatopathologists considered basal proliferation to be more important in terms of progression risk than upwards directed growth patterns. It should be considered to classify AKs according to their basal proliferation pattern (PRO I-III).
\end{abstract}

\section{Conflicts of interest}

L. Schmitz: Lecture fees from Almirall, Biofrontera, Galderma, Meda. Member of advisory boards for Almirall. G. Gupta: Lecture fees from Abbvie, Galderma, Leo and Meda. Member of advisory boards for Almirall, Leo, Meda and Novartis. M. Stücker: Lecture fees from Juzo, Medi; member of advisory boards for Eurocom, Bauerfeind. M. Doerler: None. T. Gambichler: Lecture fees and advisory boards from Merck, Novartis, Roche, NeraCare, BMS. J. Welzel: Lecture fees from Almirall, Leo, Pierre Fabre, Novartis, Galderma. RM. Szeimies: Lecture fees from Almirall, Biofrontera, Galderma, Leo, photonamic; member of advisory boards for Almirall, Biofrontera, Galderma, Leo; grants from European Commission. E. Bierhoff: None. E. Stockfleth: Lecture fees from Almirall, Leo, Pierre Fabre. T. Dirschka: Lecture fees from Almirall, Biofrontera, Galderma, Leo, Meda, Riemser, Janssen; member of advisory boards for Almirall, Biofrontera, Leo, Meda, Novartis, Riemser, Janssen; unrestricted grants from Meda and Galderma. Study fees from Schulze \& Böhm GmbH. 
None.

\section{Introduction}

Actinic keratoses (AKs) are regarded as early in-situ squamous cell carcinoma (SCC). They can progress into invasive carcinoma, subsequently metastasize and lead to a life-threatening disease. ${ }^{1-3}$ The incidence in fair skin people continues to increase due to a change in demography and leisure activities in industrial nations. AKs represent chronic disease which should be treated to control field change and to possibly prevent progression into invasive SCC. ${ }^{4}$ However, with increasing numbers of patients with AKs, social and economic issues require consideration as there would be a substantial impact on health budgets. This may necessitate risk stratification with patients at higher risk of developing invasive SCCs to be more actively treated. Hence, reliable predictive histological and clinical characteristics are required to provide individual therapy regimes for each patient.

Actinic keratoses are commonly assessed either by clinical characteristics $^{5}$ or histological criteria. ${ }^{6-8}$ In recent years, clinical evaluation of AKs has changed following two independent studies which showed that the thickness of a distinct AK lesion does not correlate with the underlying histology or p53 expression., ${ }^{9,10}$ Thus, clinical evaluation has moved from the assessment of single lesions to the assessment of the entire actinically damaged field and related features such as thickness, erythema and distribution of AK lesions. ${ }^{11}$ A recent study showed a significantly higher actinic keratosis and severity index (AKASI) in patients with invasive SCC than in patients with BCC or patients without invasive tumours, ${ }^{12}$ suggesting that invasive SCCs occurred in patients with more actinic change.

Besides clinical assessment, histology remains the gold standard in discriminating between in-situ and invasive tumours. In analogy with other intraepithelial neoplasms such as cervical intraepithelial neoplasia (CIN) or vulvar intraepithelial neoplasia (VIN), AKs have been classified according to the atypical keratinocyte distribution throughout the epidermis. However, subsequent studies have shown that this progression model is not appropriate. AK III lesions representing full thickness atypia of the epidermis were not associated with the highest risk for invasion. $^{13}$ On the contrary, AKs with atypical keratinocytes restricted to the lower third (AK I) of the epidermis as well as AKs with an advanced basal proliferation (PRO III) were more commonly associated with invasive SCCs in their direct vicinity. ${ }^{13,14}$ Thus, the assessment of basal growth pattern is important to evaluate progression risk and should be applied to histologically classify all AKs.

It is also important that independent investigators are able to apply classification schemes in the same manner and obtain agreement in the assessment of the same histology. Hence, inter-rater reliability represents a decisive criterion for the quality of a scoring system. ${ }^{15}$

This study was conducted to compare the inter-rater reliability of the established classification of upwards directed growth of AKs (AK I-III) ${ }^{7}$ and the recently established classification of basal growth pattern of AKs (PRO I-III). ${ }^{8}$ This study aimed to analyse the agreement between experienced dermatopathologists of their histological assessment and in addition their presumed risk of invasion (PRI) for both classification schemes.

\section{Materials and methods}

\section{Sample collection}

This prospective study was performed at the Skin Cancer Centre of the Ruhr-University Bochum (Bochum, Germany) and conducted according to the Declaration of Helsinki. Between June 2017 and August 2017, samples were collected from consecutive AK specimens located on the head sent for histopathological analysis. AK samples were restricted to this region to reduce histological variability and to ensure comparability of histological findings. As tumour progression is often promoted by immunosuppression, we excluded samples from patients on immunosuppressive therapy from this study.

\section{Survey preparation and design}

In total, 71 haematoxylin and eosin (H\&E) stained sections of AKs were scanned to avoid artefacts from preparation procedures and to ensure that all relevant histomorphological structures such as all epidermal layers, dermo-epidermal junction (DEJ) zone and stratum papillare were present. Shave excisions and samples with a width of $3 \mathrm{~mm}$ or less were excluded. Finally, 54 samples met all aforementioned criteria and were subsequently processed. Images of H\&E sections with an original magnification of 40 -fold were uploaded to the survey platform 'limesurvey' (www.limesurvey.org; LimeSurvey GmbH, Hamburg, Germany). The utilized area of this survey platform was password protected and not freely available.

Dermatologists and pathologists educated in dermatopathology $(n=21)$ participated. After login, a short explanation of both histological classification systems was given to harmonize and update the theoretical knowledge of all raters. The PROgrading was classified into three grades (protruding I-III) or if there was no basal proliferation, 'none' was chosen. ${ }^{8}$ PRO I (crowding) is defined by atypical keratinocytes which are crowding at basal epidermal layers resulting in a more basophilic appearance. PRO II (budding) shows small hemispheric buds starting at the basal epidermis to protrude slightly into the upper 
papillary dermis and forming round nests of atypical keratinocytes. PRO III (papillary sprouting) presents spiky or filiform papillary elongation of atypical keratinocytes protruding into the upper dermis while this protrusion exceeds the thickness of the overlying epidermis.

The classification scheme according to Röwert-Huber et al. ${ }^{7}$ was defined as follows: in AK I (mild), atypical keratinocytes are limited to the lower third of the epidermis; in AK II (moderate), atypical keratinocytes extend to the lower two-thirds of the epidermis; in AK III (severe) full thickness atypia of the epidermis is observed.

\section{Survey structure}

Following the introduction, all 54 histological images were presented to each rater on individual pages, and raters were asked to provide the AK-grading (I-III) according to Röwert-Huber (upwards directed growth) and the PRO-grading (0-III) according to the basal growth pattern (downwards directed growth). Moreover, raters could make a statement of risk (none, mild, moderate, severe, very severe) of the shown AK lesion to progress into invasive carcinoma. The rater's presumption of the underlying progression risk was defined as 'presumed risk of invasion'.

Grading (AK, PRO and PRI) of each lesion was defined by the modal value of what the 21 raters diagnosed. In other words, the overall grading of each AK lesion was ascertained by the grade that was most likely to be diagnosed.

At the end of this survey, the raters were asked to assess the scheme: (i) if it was easy to apply (fully agree, partly agree and disagree); (ii) if one of these classification schemes was more relevant in predicting the risk of $\mathrm{AK}$ lesions to progress into invasive carcinoma; (iii) and if yes, which one.

\section{Statistical analysis}

Data analysis was performed using the statistical package MedCalc software version 17.6 (Ostend, Belgium) and IBM SPSS Statistics for Windows, version 21.0 (Armonk, NY, USA: IBM Corp.). The distribution of data was assessed by the D'AgostinoPearson test. If there was normal distribution, data were expressed as mean and standard deviation (SD); if not, data were expressed as median and range. Data were statistically analysed using the Spearman's coefficient of rank correlation and the chisquared test, with $P$-values $<0.05$ being considered statistically significant. Kappa $(k)$ statistic was used to calculate inter-rater agreement for ordinal data. Therefore, Kendall's coefficient ( $W$ and tau) of concordance was analysed. Based on investigations of Landis and $\operatorname{Koch}^{16}$ the degree of agreement was evaluated according to the following ranges of $k$ : 0 poor agreement; $0-0.2$ slight agreement; 0.2-0.4 fair agreement; 0.4-0.6 moderate agreement; 0.6-0.8 substantial agreement; $0.8-1$ almost perfect agreement.

\section{Results}

The survey was completed by 21 expert dermatopathologists, thereof 12 dermatologists (57.1\%) and nine pathologists (42.9\%). The mean (standard deviation; SD) age was 43.4 $( \pm 9.6)$ years and a mean $(S D)$ experience in dermatopathology of 10.9 ( \pm 8.6 ) years. The majority of investigators histologically classified $16.7 \%(9 / 54)$ as AK I, 66.7\% (36/54) as AK II, and $16.7 \%(9 / 54)$ as AK III. With regards to basal growth pattern, $25.9 \%(14 / 54)$ were classified as PRO I, 42.6\% (23/54) as PRO II, and $31.5 \%(17 / 54)$ as PRO III. As the grading of each lesion was defined by the modal value, AK I was diagnosed by dermatopathologists with a mean (SD) of $63.5 \%( \pm 13.3)$, AK II by $65.9 \%( \pm 12.9)$, and AK III by $85.7 \%( \pm 9.5)$ as well as PRO I with a mean $(\mathrm{SD})$ of $78.6 \%( \pm 10.2)$, PRO II by $70.2 \%( \pm 12.5)$, and PRO III by $83.5 \%( \pm 12.5)$. Based on the overall impression of the AK histology, the progression risk into invasive carcinoma was evaluated in $18.5 \%(10 / 54)$ as mild, in $46.3 \%(25 / 54)$ as moderate, in $33.3 \%(18 / 54)$ as severe, and in $1.9 \%(1 / 54)$ as very severe (Fig. 1).

Kendall's $W$ coefficient showed that throughout all $54 \mathrm{AK}$ lesions there was moderate inter-rater reliability $(k=0.488)$ of AK I-III classification (upwards directed growth) which was highly significant $(P<0.001)$. The PRO I-III classification (basal proliferation) showed a higher 'substantial' inter-rater reliability ( $k=0.793$ ) which was very close to perfect reliability (defined as $\geq 0.8$ ) with $P<0.001$. With respect to both classifications, the evaluation of PRI showed highly significant moderate inter-rater reliability (0.562) (Fig. 2). Examples of agreements and disagreements by raters are shown in Fig. 3.

Kendall's coefficient showed a highly significant concordance to PRI for both classifications $(P<0.001)$. PRI showed a Kendall's coefficient of 0.563 for AK classification (0.4-0.6: moderate agreement) and 0.759 for PRO classification (0.6-0.8: substantial

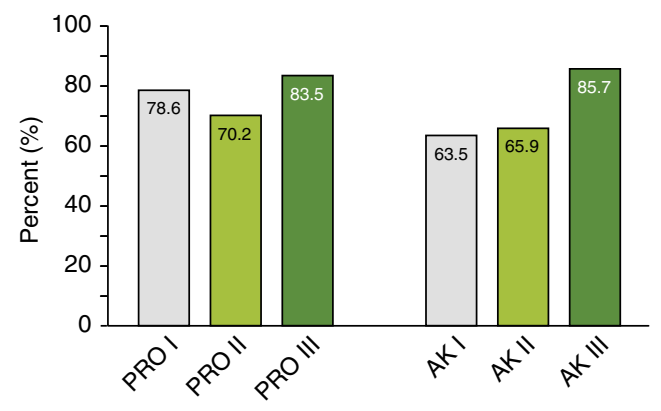

Figure 1 Mean agreement of each grading by the 21 investigators. Grading of each lesion was defined by what the investigators mostly diagnosed (mode). Discriminatory power of gradings within the PRO classification was statistically significant between PRO I/ III and PRO II $(P<0.005)$ and in AK classification between AK I/II and AK III $(P<0.0001)$. 


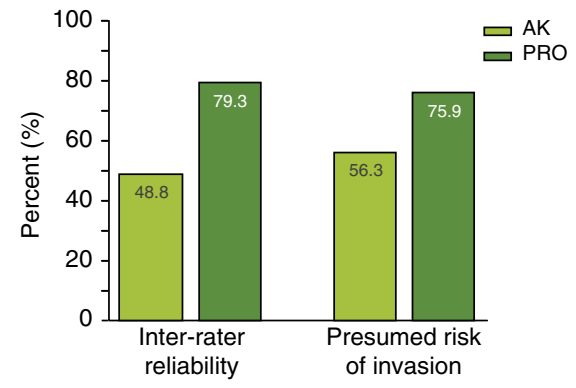

Figure 2 Inter-rater reliability and presumed risk of invasion using both histological classifications by 21 dermatopathologists and 54 AK lesions. Moderate agreement was seen with the AK classification scheme (0.488) compared to substantial agreement (0.793) close to almost perfect agreement $(>0.800)$ with the PRO classification. Agreement according to presumed risk of invasion (PRI) in terms of validity was moderate (0.563) for the AK classification scheme compared to substantial agreement $(0.759)$ for PRO classification. Each value was highly statistical significant $(P<0.001)$.
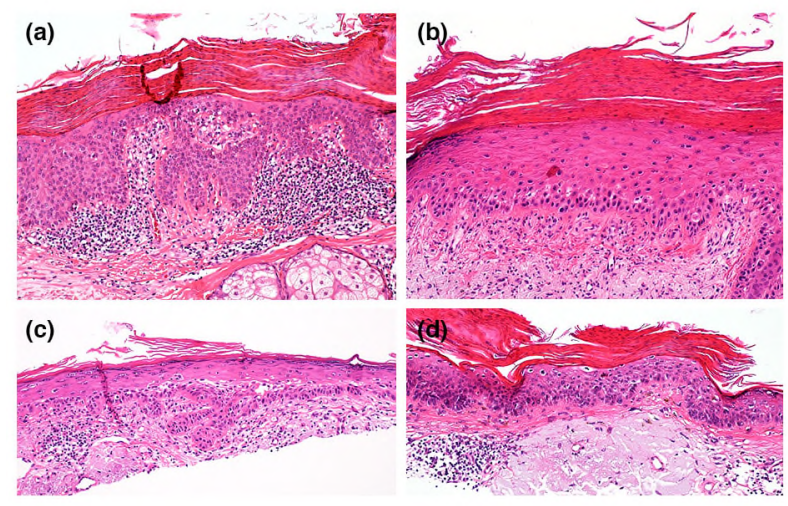

Figure 3 Example of four AK lesions (H\&E sections, original magnification $\times 40$ ), which were part of the survey and showed different evaluations by the 21 dermatopathologists. (a) This lesion showed a very high agreement of the diagnosed gradings [AK III (0.952), PRO III (1.0), and presumed risk of invasion (PRI) III (0.714)]. (b) In contrast to (a), this lesion showed a poor agreement among the investigators [AK I (0.571), PRO II (0.524), and PRI II (0.524)]. (c) This lesion showed a high agreement for PRO classification [PRO III (0.952)] in contrast to AK grading [AK II (0.667)]. (d) The shown lesion showed the opposite with high agreement for AK classification [AK III (0.905)] compared to PRO classification [PRO II (0.667)].

agreement). There was, therefore, higher agreement amongst raters of an association between PRO classification and progression risk than the $\mathrm{AK}$ classification. Details are shown in Table 1.

After completing the histological classification of all $54 \mathrm{AK}$ samples, the participants were asked, if they considered the
Table 1 Kendall's coefficient of concordance for both classifications of each investigator regarding their presumed invasion risk based on the evaluation of all 54 histological images

\begin{tabular}{|c|c|c|}
\hline \multirow[t]{2}{*}{ Rater } & \multicolumn{2}{|c|}{$\begin{array}{l}\text { Kendall's coefficient of concordance for } \\
\text { both classifications compared to presumed } \\
\text { risk of invasion (PRI) }\end{array}$} \\
\hline & AK vs. PRI & PRO vs. PRI \\
\hline 1 & $0.471^{\star \star}$ & $0.810^{\star \star}$ \\
\hline 2 & $0.278^{*}$ & $0.743^{\star \star}$ \\
\hline 3 & $0.279^{*}$ & $0.797^{\star \star}$ \\
\hline 4 & $0.461^{\star *}$ & $0.706^{* *}$ \\
\hline 5 & $0.609^{* *}$ & $0.779^{\star \star}$ \\
\hline 6 & $0.286^{*}$ & $0.723^{\star \star}$ \\
\hline 7 & $0.365^{\star \star}$ & $0.603^{\star *}$ \\
\hline 8 & $0.512^{\star \star}$ & $0.612^{\star *}$ \\
\hline 9 & $0.568^{\star *}$ & $0.662^{\star \star}$ \\
\hline 10 & $0.712^{* *}$ & $0.500^{* *}$ \\
\hline 11 & $0.339^{\star \star}$ & $0.326^{* *}$ \\
\hline 12 & $0.507^{\star *}$ & $0.652^{* \star}$ \\
\hline 13 & $0.359^{\star *}$ & $0.712^{* \star}$ \\
\hline 14 & 0.183 & $0.445^{\star \star}$ \\
\hline 15 & $0.685^{\star \star}$ & $0.442^{* *}$ \\
\hline 16 & $0.450^{\star *}$ & $0.527^{\star \star}$ \\
\hline 17 & $0.424^{\star \star}$ & $0.386^{\star \star}$ \\
\hline 18 & $0.476^{\star \star}$ & $0.602^{\star *}$ \\
\hline 19 & $0.492^{* *}$ & $0.659^{\star \star}$ \\
\hline 20 & $0.378^{\star \star}$ & $0.831^{* *}$ \\
\hline 21 & $0.473^{\star *}$ & $0.619^{\star \star}$ \\
\hline Overall & $0.563^{\star \star}$ & $0.759^{\star *}$ \\
\hline
\end{tabular}

$* P<0.05 . * * P<0.01$.

$k$, indicates degree of agreement: 0 poor agreement; 0-0.2 slight agreement; 0.2-0.4 fair agreement; 0.4-0.6 moderate agreement; 0.6-0.8 substantial agreement; 0.8-1 almost perfect agreement.

application of each score as easy. In case of Röwert-Huber grading system (AK I-III), 61.9\% (13/21) fully agreed, 33.3\% (7/21) partly agreed, and $4.8 \%(1 / 21)$ disagreed. With respect to basal growth grading system (PRO I-III), 85.7\% (18/21) fully agreed, $14.3 \%$ partly, and no one disagreed. Finally, they were asked, which of the classification schemes was more relevant in predicting the risk of $\mathrm{AK}$ lesions progressing into invasive carcinoma. One investigator $(4.8 \%)$ considered the AK classification was more relevant, fourteen $(66.7 \%)$ the basal growth depended classification, and six $(28.6 \%)$ thought both classifications were equivalent.

\section{Discussion}

Most medical classifications are expected to meet two main requirements: firstly, the degree to which it measures what it is supposed to measure and secondly the agreement achieved amongst raters. ${ }^{15}$ In terms of classifying AKs, validity should represent the risk of AKs progressing into invasive carcinoma. Our study showed a high and almost perfect inter-rater 
reliability of the PRO classification compared to a moderate inter-rater reliability of the common AK classification. Thus, dermatopathologists should be able to provide better concordant gradings of AK lesions according to their basal growth pattern to clinicians.

Regarding dermatopathologists' estimation of progression risk, we obtained a moderate agreement for the common AK classification compared to a high agreement on the PRO classification. PRI is a subjective prognostic measure of the grading schemes. It is, however, open to bias without a prospective approach as it is difficult to conclude whether the AK progressed to invasive SCC or what happened to skin adjacent to excised tissue. Moreover, this study was carried out prior to another recently published study which showed a significant association between basal proliferating AKs and invasive SCCs. ${ }^{14}$ Thus, the dermatopathologists were not aware of these results and subsequently not influenced by them, so that their conclusions were purely based on their dermatohistological experience. Analysing results from both studies, basal proliferation and thus PRO classification provides a much better risk stratification in terms of progression risk than common AK classifications. The believed hypothesis of a disease continuum analogous to CIN is not applicable for keratinocytic intraepidermal neoplasia. Reporting on these dermatopathological findings alone, clinicians may incorrectly under or over treat their patients. It is, therefore, not reasonable that the term 'carcinoma in-situ' is restricted merely to AKs showing full thickness epithelial atypia. AK classifications based on the distribution of atypical keratinocytes throughout epidermal layers should be abandoned.

Gradings obtained were variably distributed in the two classifications schemes. Within the common AK classification, the intermediate grading (AK II) of this three-tiered classification was diagnosed at most in two-thirds of lesions compared to 16.7\% each for AK I and AK III. On the one hand, this may represent the true histological distribution or it could be that this grade may lack discriminatory power. Figure 1 shows mean concordance of each grading (AK I-III) by the investigators. Besides AK III, concordance in AK I and AK II amongst the investigators was quite low, which indicates a low discriminatory power compared to AK III grading. This might be based on the fact that many dermatopathologists might falsely think of a carcinoma in-situ and full thickness atypia to fulfil the criterion for AK III. Interestingly, the AK classification according to Röwert-Huber et al. ${ }^{7}$ is not analogous to the 'KIN' classification by Cockerell et al. ${ }^{6}$ Besides missing clinical criteria in Röwert-Huber's AK classification, there are some clear differences in classifying each grade. For example, KIN III represents full thickness atypia in contrast to AK III which includes AK lesions with atypical keratinocytes throughout the last third of epidermal layers.

In contrast, the PRO classification shows higher concordance among PRO I and PRO III grading. Intermediate PRO II grading showed lowest concordance indicating that PRO I and III are easier to define by dermatopathologists. It might be advantageous that both highest and lowest grading are diagnosed with a higher concordance in terms of reliable risk stratification with lower and higher risks of progression.

The results are mirrored by the investigators' views with most stating that the PRO classification was easier to apply than AK classification. With regards to the question of which scheme was more relevant in predicting progression risk, two-thirds thought the PRO classification was more relevant compared to one investigator who chose the AK classification.

Further to these scores, there are different morphological subtypes of AKs such as acantholytic or bowenoid which are also used to characterize a lesion. Until now, there is no uniform consensus of what histological information on AK lesions should be provided by dermatopathologists to clinicians, particularly features which may give prognostic value as this is what clinicians require to decide on further management. With current evidence this prognostic information should focus on atypical keratinocytes along the basement membrane and their associated basal proliferation. ${ }^{13,14,17,18}$ Thus, a histological report should answer two main questions: (i) Invasive or in-situ? (ii) Grade of basal proliferation? Additional information may not be helpful in providing prognostic detail as evidence for this is lacking.

Additionally, we intentionally choose representative images with a uniform appearance. This study should evaluate the applicability and avoid bias as much as possible. In daily practice, however, most AKs present several grades within one lesion at the same time. ${ }^{19}$ Pivotal publications of both scores have shown that an AK should be classified according to the highest underlying grade within a lesion. ${ }^{7,8}$ Nevertheless, on which basis entire AK lesions should be classified (highest grade, even it presents only a small part of the AK, in contrast to the overall impression of a lesion) is another important issue and still remains controversial. It should be addressed in further research due to what criteria AK lesions should be classified.

The small number of dermatopathologists who have participated could be a limitation of our study. Nevertheless, these were 21 experienced dermatopathologists who evaluated $54 \mathrm{AK}$ lesions and so should have provided reasonable and useful data. Additionally, it could have been helpful to provide the answer opurtunity 'early invasive SCC' as it is sometimes very difficult or even impossible to discriminate between AK and early SCC. However, we did not achieve any responses of participating centres that SCC was considered to be the assumed diagnosis. Moreover, assessing AK lesions adjacent to invasive SCC may have been a better approach to overcome the question of actual progression risk of each lesion.

In conclusion, high inter-rater reliability of the PRO classification in predicting progression risk along with data on its association with invasive SCCs favours its routine use in the pathological reports of AKs. 


\section{References}

1 Ackerman AB, Mones JM. Solar (actinic) keratosis is squamous cell carcinoma. Br J Dermatol 2006; 155: 9-22.

2 Salasche SJ. Epidemiology of actinic keratoses and squamous cell carcinoma. J Am Acad Dermatol 2000; 42: 4-7.

3 Stockfleth E, Ferrandiz C, Grob JJ et al. Development of a treatment algorithm for actinic keratoses: a European Consensus. Eur J Dermatol 2008 ; 18: 651-659.

4 Dirschka T, Gupta G, Micali G et al. Real-world approach to actinic keratosis management: practical treatment algorithm for office-based dermatology. J Dermatolog Treat 2017; 28: 431-442.

5 Olsen EA, Abernethy ML, Kulp-Shorten C et al. A double-blind, vehicle-controlled study evaluating masoprocol cream in the treatment of actinic keratoses on the head and neck. J Am Acad Dermatol 1991; 24: 738-743.

6 Cockerell CJ. Histopathology of incipient intraepidermal squamous cell carcinoma ("actinic keratosis"). J Am Acad Dermatol 2000; 42: 11-17.

7 Rowert-Huber J, Patel MJ, Forschner T et al. Actinic keratosis is an early in situ squamous cell carcinoma: a proposal for reclassification. $\mathrm{Br} J \mathrm{Der}$ matol 2007; 156(Suppl 3): 8-12.

8 Schmitz L, Gambichler T, Gupta G et al. Actinic keratoses show variable histological basal growth patterns - a proposed classification adjustment. J Eur Acad Dermatol Venereol 2018; 32: 745-751.

9 Heerfordt IM, Nissen CV, Poulsen T, Philipsen PA, Wulf HC. Thickness of actinic keratosis does not predict dysplasia severity or P53 expression. Sci Rep 2016; 6: 33952.

10 Schmitz L, Kahl P, Majores M, Bierhoff E, Stockfleth E, Dirschka T. Actinic keratosis: correlation between clinical and histological classification systems. J Eur Acad Dermatol Venereol 2016; 30: 1303-1307.
11 Dirschka T, Pellacani G, Micali G et al. A proposed scoring system for assessing the severity of actinic keratosis on the head: actinic keratosis area and severity index. J Eur Acad Dermatol Venereol 2017; 31: 12951302.

12 Schmitz L, Gambichler T, Gupta G, Stucker M, Dirschka T. Actinic keratosis area and severity index (AKASI) is associated with the incidence of squamous cell carcinoma. J Eur Acad Dermatol Venereol 2018; 32: 752756.

13 Fernandez-Figueras MT, Carrato C, Saenz X et al. Actinic keratosis with atypical basal cells (AK I) is the most common lesion associated with invasive squamous cell carcinoma of the skin. J Eur Acad Dermatol Venereol 2015; 29: 991-997.

14 Schmitz L, Gambichler T, Kost C et al. Cutaneous squamous cell carcinomas are associated with basal proliferating actinic keratoses. $\mathrm{Br} J$ Dermatol 2018. https://doi.org/10.1111/bjd.16536

$15 \mathrm{McHugh}$ ML. Interrater reliability: the kappa statistic. Biochem Med (Zagreb) 2012; 22: 276-282.

16 Landis JR, Koch GG. The measurement of observer agreement for categorical data. Biometrics 1977; 33: 159-174.

17 Fernandez Figueras MT. From actinic keratosis to squamous cell carcinoma: pathophysiology revisited. J Eur Acad Dermatol Venereol 2017; 31 (Suppl 2): 5-7.

18 Saenz-Sarda X, Carrato C, Perez-Roca L et al. Epithelial-to-mesenchymal transition contributes to invasion in squamous cell carcinomas originated from actinic keratosis through the differentiated pathway, whereas proliferation plays a more significant role in the classical pathway. J Eur Acad Dermatol Venereol 2018; 32: 581-586.

19 Schmitz L, Stucker M, Gambichler T, Stockfleth E, Dirschka T. Histological intralesional heterogeneity of actinic keratoses relates to field cancerization. J Dtsch Dermatol Ges 2018; 16: 1211-1217. 